\title{
$\pi$-Conjugated Small Molecules Enable Efficient Perovskite Growth and Charge-Extraction for High-Performance Photovoltaic Devices
}

\author{
Hui Liu ${ }^{a}$, Hai-Rui Liu ${ }^{\mathrm{a}, *}$, Feng Yang ${ }^{\mathrm{a}}$, Ji-En Yanga ${ }^{\mathrm{a}}$, Jian Song ${ }^{\mathrm{a}}$, Meng Li ${ }^{\mathrm{b}, \mathrm{c}, *}$, Zhe Lid, \\ Wing Chung Tsoi ${ }^{\mathrm{e}}$, Chinweokwu Eze Martin ${ }^{\mathrm{d}}$, Zhi-Yong Liu ${ }^{\mathrm{a}}$, Heng Ma ${ }^{\mathrm{a}}$, Min Gao ${ }^{\mathrm{d}}$, \\ Zhao-Kui Wang ${ }^{\text {b** }}$ \\ ${ }^{a}$ Henan Key Laboratory of Photovoltaic Materials, College of Physics \& Materials Science, \\ Henan Normal University, Xinxiang 453007, China. \\ ${ }^{b}$ Institute of Functional Nano \& Soft Materials (FUNSOM), Jiangsu Key Laboratory for \\ Carbon-Based Functional Materials \& Devices, Soochow University, Suzhou 215123, China. \\ ${ }^{c}$ Helmholtz-Zentrum Berlin für Materialien und Energie, Kekuléstraße 5, 12489 Berlin, Germany, \\ Laboratory of Advanced Optoelectronic Materials. \\ ${ }^{d}$ School of Engineering, Cardiff University, Cardiff, Wales CF24 3AA, UK \\ ${ }^{e}$ College of Engineering, Swansea University Bay Campus, Fabian Way SAl 8EN, Swansea, UK
}

\section{Abstract}

A

$\pi$-conjugated

small

molecule

N,N'-bis(naphthalen-1-yl)-N,N'+-bis(phenyl)benzidine (NPB) is introduced into a poly (bis(4-phenyl)(2,4,6-trimethylphenyl)amine) (PTAA) hole transport layer in inverted perovskite solar cells (PSCs). The NPB doping induces better perovskite crystal growth owing to a strong $\pi-\pi$ interaction with PTAA and cation- $\pi$ interactions with $\mathrm{CH}_{3} \mathrm{NH}_{3}{ }^{+}\left(\mathrm{MA}^{+}\right)$. In addition, NPB doping not only improves the wettability of PTAA and regulates the perovskite crystallization to achieve a larger grain size, but also moves the valence band energy of the hole transport layer closer to the perovskite layer. Consequently, the fabricated PSCs delivered a power conversion efficiency (PCE) of $20.15 \%$, with a short-circuit current density $\left(J_{S C}\right)$ of $22.60 \mathrm{~mA} / \mathrm{cm}^{2}$ and 
open-circuit voltage $\left(V_{O C}\right)$ of $1.14 \mathrm{~V}$. This outcome indicates that PTAA:NPB composite materials present great potential for fabricating high-performance PSCs.

Keywords: $\pi$-conjugated molecule; Energy level alignment; Charge extraction

\section{Introduction}

Organometallic trihalide perovskite solar cells (PSCs) have captured enormous attention worldwide for their unprecedented developments in the past few years, with a highest certified power conversion efficiency (PCE) of $25.2 \%$ up till now [1]. To improve the performance of PSCs, various novel device architectures have been developed [2-4], among which planar inverted PSCs have shown great potential owing to their negligible hysteresis, low-temperature processability, and simple device fabrication [5-8].

A key step in the fabrication of planar inverted PSCs is the deposition of a perovskite absorber layer on top of a hole transport layer (HTL), whereby the HTL can significantly influence the perovskite absorber layer deposition and crystallization. To date, various types of hole transport materials have been developed and employed in high efficiency PSCs. For example, p-type inorganic materials including copper compounds $[9,10]$, nickel oxide $(\mathrm{NiO})$ [11] and vanadium oxide $\left(\mathrm{V}_{2} \mathrm{O}_{5}\right)$ [12], have been employed as an HTL in inverted PSCs for their high chemical stability and hole mobility. However, the fabrication of devices based on these materials still require high-temperature sintering process or vacuum-process. To overcome these drawbacks, poly (3,4-ethylenedioxythiopene):poly (styrenesulfonic acid) (PEDOT:PSS) has been employed in the inverted planar PSCs as an organic HTL material [13,14]. 
Unfortunately, the energy level mismatch between PEDOT:PSS (-4.9 to $-5.1 \mathrm{eV}$ ) and $\mathrm{CH}_{3} \mathrm{NH}_{3} \mathrm{PbI}_{3}(-5.4 \mathrm{eV})$ can result in relatively low open-circuit voltages $\left(V_{O C}\right)$, therefore, only a modest device performance $[15,16]$. Furthermore, the acidity and hydrophilicity of PEDOT:PSS has detrimental effects on device performance and stability [17,18]. Poly (bis(4-phenyl)(2,4,6-trimethylphenyl)amine) (PTAA), an organic p-type semiconductor that has been previously used in organic electronics and photovoltaics $[19,20]$, has recently been established as a promising HTL material for PSCs in both n-i-p and p-i-n configurations [21,22]. The relatively large energy gap between the highest occupied molecular orbital (HOMO) energy level of PTAA (-5.1 $\mathrm{eV}$ ) and the valence band maximums (VBMs) of pristine perovskite provides substantial potential to maximize the $V_{O C}$ of PSCs by manipulating the HOMO energy level of PTAA closer to the VBMs of the perovskite materials [21]. Yet, the hydrophobic nature of PTAA makes it difficult to spin coat the precursor solution on top of HTL. To overcome this limitation, oxygen plasma and ultraviolet-ozone treatment are typically adopted to improve the wettability of numerous hydrophobic HTLs $[19,23,24]$. However, these treatments can cause degradation of the organic HTL materials, because they changed the chemical bond of the molecule, including the chemical bond category and binding energy, then the nature of material will be altered to some extent like the hydrophily and stability. And it is furthermore difficult to control the processing conditions (e.g. power, processing time), resulting in poor reproducibility. Lee et al. introduced an amphiphilic molecule poly [(9,9-bis (3'(N,N-dimethylamino) propyl) -2,7-fluorene) -alt 2,7- (9,9-dioctylfluorene)] (PFN) to 
serve as a connected bridge between hydrophobic PTAA and perovskite layer [25]. 2,3,5,6 Tetrafluoro-7,7,8,8-tetracyanoquinodimethane (F4-TCNQ) has been doped into PTAA to improve the conductivity of PTAA HTLs to further increase the $V_{O C}$ and device efficiency [26]. While, F4-TCNQ doped in PTAA forms reactive sites for charge accumulation, which renders an improvement in device performance [27,28]. A high PCE of $20.75 \%$ was obtained for inverted planar PSCs based on PTAA material that was doped with F4-TCNQ and plasma treated, and introduced of a PMMA interfacial layer at the same time, but this process is rather complicated [27]. To date, a systematic study for the engineering of PTAA HTLs to simultaneously optimize their hydrophilicity and improve the performance of PSCs is still lacking.

N,N'-bis(naphthalen-1-yl)-N,N'+-bis(phenyl)benzidine (NPB), consisting of triphenylamine units and a $\pi$-conjugated structure, has been used as an interlayer material in inorganic light-emitting diodes, owing to its excellent semiconducting properties, superior electrical and optical properties, and modest hole mobility [29-31]. Hence, Li et al. utilized NPB as an HTL in inverted PSCs and achieved a PCE of $19.96 \%$ [32]. As discussed above, very few works have investigated the use of PTAA and NPB as an HTL material in PSCs to date. Especially, there is an urgent need to solve the limitation of PTAA.

Herein, high performance inverted planar PSCs based on HTLs comprised of NPB-doping into PTAA films are reported. NPB-doping not only increases the wettability of PTAA HTL but also regulates the quality of the subsequently-deposited perovskite films. To elucidate the impact of NPB-doping (through composition 
engineering) of the PTAA HTL, optical, electrical, and structural properties of PTAA and PTAA:NPB HTLs were systematically studied. NPB-doping of PTAA promotes the extraction and transportation of holes from the perovskite absorber layer to the PTAA:NPB HTL, leading to a significantly improved fill factor (FF). PTAA:NPB-based devices possess a higher built-in potential, resulting in reduced recombination losses, and hence enhanced $V_{O C}$. Devices with PTAA:NPB as an HTL show a PCE of $20.15 \%$ compared to $17.92 \%$ for a reference device. Furthermore, devices based on NPB-doping PTAA HTLs showed negligible hysteresis in the current density-voltage $(J-V)$ characteristics.

\section{Experimental Section}

\subsection{Materials preparation}

Methylammonium iodide (MAI) was purchased from Dyesol. Organic solvents including anhydrous N, N-dimethyl formamide (DMF), dimethyl sulfoxide (DMSO) and chlorobenzene (CB) were purchased from Sigma-Aldrich. Unless otherwise indicated, all materials were obtained from Xi'an Polymer Light Technology Corp. The PTAA $(0.5 \mathrm{mg} / \mathrm{mL})$ and NPB $(2.5 \mathrm{mg} / \mathrm{mL})$ were dissolved in chlorobenzene, and then mixed using desired ratios for NPB/PTAA ranging from $0.1,0.5,1.0,1.5$ to 3.0 wt\%. Low NPB/PTAA doping ratios were obtained by diluting the high doping solution, and all mixed solutions were stirred and heated at $70{ }^{\circ} \mathrm{C}$ overnight. The perovskite precursor solution contained $645 \mathrm{mg}$ of $\mathrm{PbI}_{2}$ and $222 \mathrm{mg}$ of $\mathrm{CH}_{3} \mathrm{NH}_{3} \mathrm{I}$ in a $1 \mathrm{~mL}$ of mixed solvent of DMSO and DMF ( $\left.\mathrm{V}_{\mathrm{DMSO}} / \mathrm{V}_{\mathrm{DMF}}=1: 4\right)$. In addition, $20 \mathrm{mg}$ of

$\mathrm{PC}_{61} \mathrm{BM}$ was dissolved in $1 \mathrm{~mL}$ of chlorobenzene. All solutions mentioned above 
were stirred at $60{ }^{\circ} \mathrm{C}$ overnight and filtered with $0.45 \mu \mathrm{m}$ nylon filters before device fabrication.

\subsection{Device fabrication}

Devices were fabricated with a configuration of ITO/ $\mathrm{HTL} / \mathrm{CH}_{3} \mathrm{NH}_{3} \mathrm{PbI}_{3} / \mathrm{PC}_{61} \mathrm{BM} / \mathrm{Ag}$. ITO (15 $\left.\Omega / s q\right)$ glass substrates were cleaned sequentially with detergent, deionized water, acetone, and ethanol under sonication for 20 minutes at $40{ }^{\circ} \mathrm{C}$. After drying, the substrates were further treated with ultraviolet-ozone for 15 minutes. HTLs with different NPB doping ratios were fabricated by spin-coating at $4000 \mathrm{rpm}$ and $3000 \mathrm{rpm}$ for $35 \mathrm{~s}$, followed annealing at $110{ }^{\circ} \mathrm{C}$ for $10 \mathrm{~min}$. For the reference devices, the substrates with pristine PTAA were transferred out of the glovebox for a $10 \mathrm{~s}$ of oxygen plasma treatment and then transferred back into the glovebox for device fabrication. Note that because the bare PTAA film was so smooth and non-wetting towards DMF and DMSO, the precursor solution hardly coated the substrate, making it difficult to fabricate good quality of perovskite film with large grain size and less grain boundary on the top of it. $\mathrm{CH}_{3} \mathrm{NH}_{3} \mathrm{PbI}_{3}$ perovskite layers were then deposited using a one-step chlorobenzene-assisted solution process. The perovskite solution was added on top of the HTL and spin-coated at $6000 \mathrm{rpm}$ for $20 \mathrm{~s}$. Then, $60 \mu \mathrm{L}$ of chlorobenzene was added dropwise at $6 \mathrm{~s}$ to form a transparent perovskite film. After $10 \mathrm{~min}$ of annealing at $100{ }^{\circ} \mathrm{C}$ on a hot plate, the perovskite film was obtained. After depositing the perovskite layer, a $\mathrm{PC}_{61} \mathrm{BM}$ electron transport layer was then deposited by spin coating at $2000 \mathrm{rpm}$ for $40 \mathrm{~s}$, followed by annealing at $60{ }^{\circ} \mathrm{C}$ for $10 \mathrm{~min}$. Finally, the 
samples were transferred to a vacuum chamber for evaporation of the silver electrodes, silver electrodes were finally deposited onto $\mathrm{PC}_{61} \mathrm{BM}$ under high vacuum through a shadow mask, defining a device area of $6.25 \mathrm{~mm}^{2}$.

\subsection{Characterization and Measurement}

X-ray diffraction (XRD) data were collected using a Panalytical X'Pert Pro X-ray Powder Diffractometer with $\mathrm{Cu} \mathrm{K} \alpha$ radiation $(\lambda=0.154 \mathrm{~nm})$. The absorption spectra for the perovskite films were measured with a Varian 5E UV/Vis/NIR spectrophotometer. Scanning electron microscopy (SEM) was performed with field-emission electrons using a Nova 230 Nano SEM to obtain both top-view and cross-sectional images of films and devices. The contact angles of modified HTLs were measured using a JC000DI optical contact-measuring system (Zhong Chen. Shang Hai). The energy levels of different modified layers were measured by Ultraviolet Photoelectron Spectroscopy (UPS, Escalab 250Xi, Thermo Scientific). Photoluminescence (PL) measurements, including steady-state PL and time-resolved PL (TRPL) spectra for perovskite films deposited on different HTLs were collected using a Horiba Jobin-Yvon LabRAM HR800 with an excitation wavelength of 480 nm. Atomic Force Microscope (AFM) images were obtained using a Veeco Multimode V instrument to evaluate the surface morphology of films in tapping and intelligent modes.

The current density-voltage characteristics of devices were measured at each $20 \mathrm{mV}$ voltage step. And the measurement is simulated under Air-Mass (AM) 1.5 sunlight at $100 \mathrm{~mW} / \mathrm{cm}^{2}$ (Newport, Class AAA solar simulator, 94023A-U) with a 2400 Series 
Source Meter (Keithley Instruments). The incident light intensity was varied from 100 $\mathrm{mW} / \mathrm{cm}^{2}$ (1 Sun) to $1 \mathrm{~mW} / \mathrm{cm}^{2}$ (0.01 Sun) and calibrated with an NREL certified KG5 filtered Si reference diode. External quantum efficiency (EQE) measurements were performed using a system combining a xenon lamp, monochromator, chopper, and lock-in amplifier and calibrated silicon photodetector. Long-term stability measurements were carried out for the best performing cells without encapsulation, the devices were kept in a dry room with controlled humidity $(20 \%<\mathrm{RH}<50 \%)$ and covered with aluminum foil during device storage. Electrical Impedance Spectroscopy (EIS) and Mott-Schottky capacitance analysis was carried out using an Ivium Electrochemical Workstation (Netherlands) under dark conditions. The dielectric spectra were tested using a Precision Impedance Analyzer (Agilent 4294A).

\section{Results and Discussion}

PSCs were fabricated with a configuration of ITO/ $\mathrm{HTL} / \mathrm{CH}_{3} \mathrm{NH}_{3} \mathrm{PbI}_{3} / \mathrm{PC}_{61} \mathrm{BM} / \mathrm{Ag}$, as shown in Fig. 1a, and the chemical structures of PTAA and NPB are shown in Fig. S1a. Gauss calculations (see Table S1 and Fig. S1b) show that the $\pi$-conjugated polymer structure of NPB has stronger $\pi-\pi$ interactions with PTAA (interaction energy $-21.0 \mathrm{kcal} / \mathrm{mol}$, structure1). In addition, NPB forms a stable molecular structure with $\mathrm{MA}^{+}$through two $\mathrm{N}-\mathrm{H}$ bonds with a bond length of $1.04 \AA$, which hooks the central tetracene moiety through $\sigma_{\mathrm{N}-\mathrm{H}}-\pi_{\mathrm{NPB}}$ interactions. The $\mathrm{NPB}$ and $\mathrm{MA}^{+}$cations have a relatively larger interaction energy of $-39.2 \mathrm{kcal} / \mathrm{mol}$ (locus 6\#), and the interaction energy between $\mathrm{MA}^{+}$and the benzene of NPB is -18.8 kcal/mol, as shown in Fig. S1c. Fig. 1b shows the image of an electrostatic potential 
(ESP) surface computed using a density functional theory (DFT) method. NPB displays a $\pi$-conjugated main chain with a dominate continuous positive ESP. Therefore, NPB can anchor $\mathrm{MA}^{+}$through stronger cation- $\pi$ interactions and coordinate between the Lewis acid and base to further improve the crystallization of the perovskite film and stability of corresponding devices, since photo-induced cation movement substantially influences device stability negatively [32,33]. A schematic diagram of the growth of $\mathrm{MAPbI}_{3}$ crystals on PTAA:NPB surface is shown in Fig. 1c.

(a)

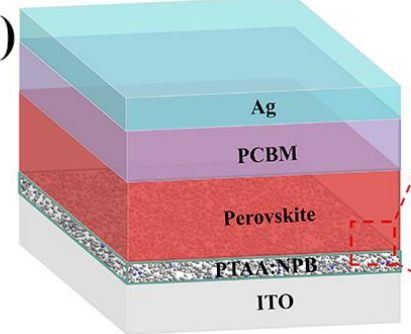

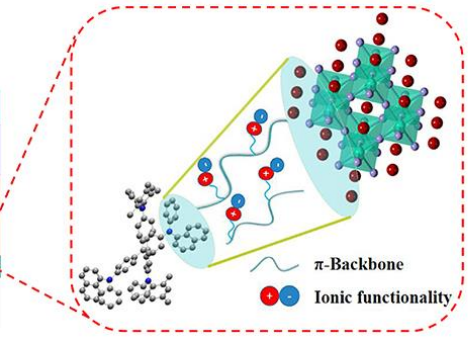

(b) $\because \underset{\mathrm{H}}{\mathrm{N}}$

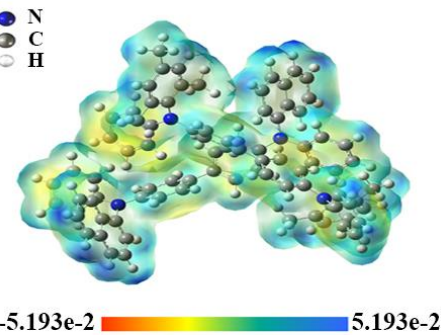

(c)

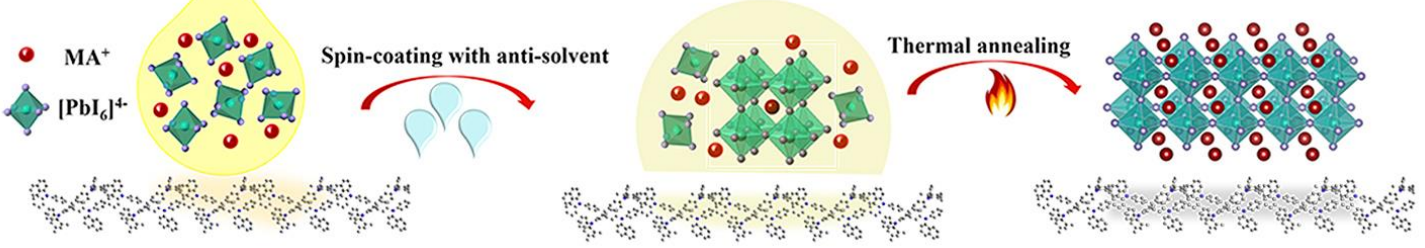

(d)

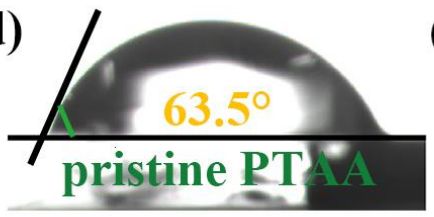

(e)

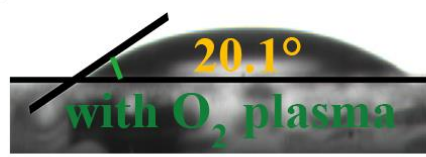

(f)

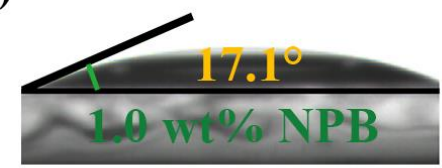

Fig. 1. Device structure of a PTAA:NPB-based p-i-n structure perovskite solar cell and the interaction between PTAA:NPB and $\mathrm{MAPbI}_{3}$. (b) ESP of PTAA and NPB. (c) Schematic diagram of the growth of $\mathrm{MAPbI}_{3}$ crystals on the PTAA:NPB surface. (d-f) DMF/DMSO component solvent contact angles for different HTL films: as-deposited pristine PTAA, pristine PTAA with oxygen plasma treatment, and PTAA with 1.0 wt\% of NPB doping, respectively.

Perovskite deposition and crystal formation processes can be affected by several HTL parameters such as chemical heterogeneities, surface roughness, and molecular 
rearrangement $[19,34]$. Therefore, the effect of NPB doping in PTAA on the surface energy of the corresponding HTLs was researched via contact angle measurements. Fig. 1d-f show the DMF/DMSO contact angles for PTAA with different treatments on glass. The as-deposited pristine PTAA shows a contact angle greater than $60^{\circ}$. While it can be clearly seen in Fig. 1e that for pristine PTAA with oxygen plasma treatment the contact angle decreased substantially to approximately $20^{\circ}$. For $1.0 \mathrm{wt} \%$ NPB-doping PTAA films, the contact angle decreased to $17.1^{\circ}$, indicating a similar effect to oxygen plasma treatment. Furthermore, the contact angles are found to vary between $32.7^{\circ}$ to $7.2^{\circ}$ for varying NPB doping between $0.1 \mathrm{wt} \%$ and $3.0 \mathrm{wt} \%$ (Fig. S2), revealing that the surface energy of PTAA can be adjusted through doping NPB. The significant decrease in contact angle is likely to contribute to the flow and spread of the precursor solution on the PTAA films, this contribution is favorable towards improved crystallinity and homogeneity for the perovskite film.

(a) Root Mean Square $2.34 \mathrm{~nm}$
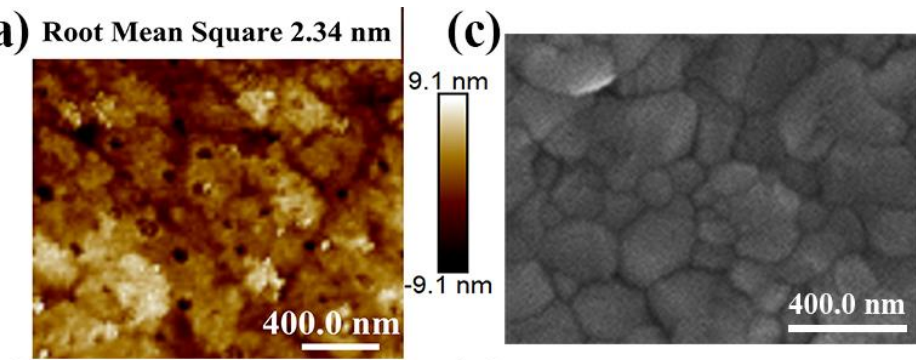

(e)

(b)
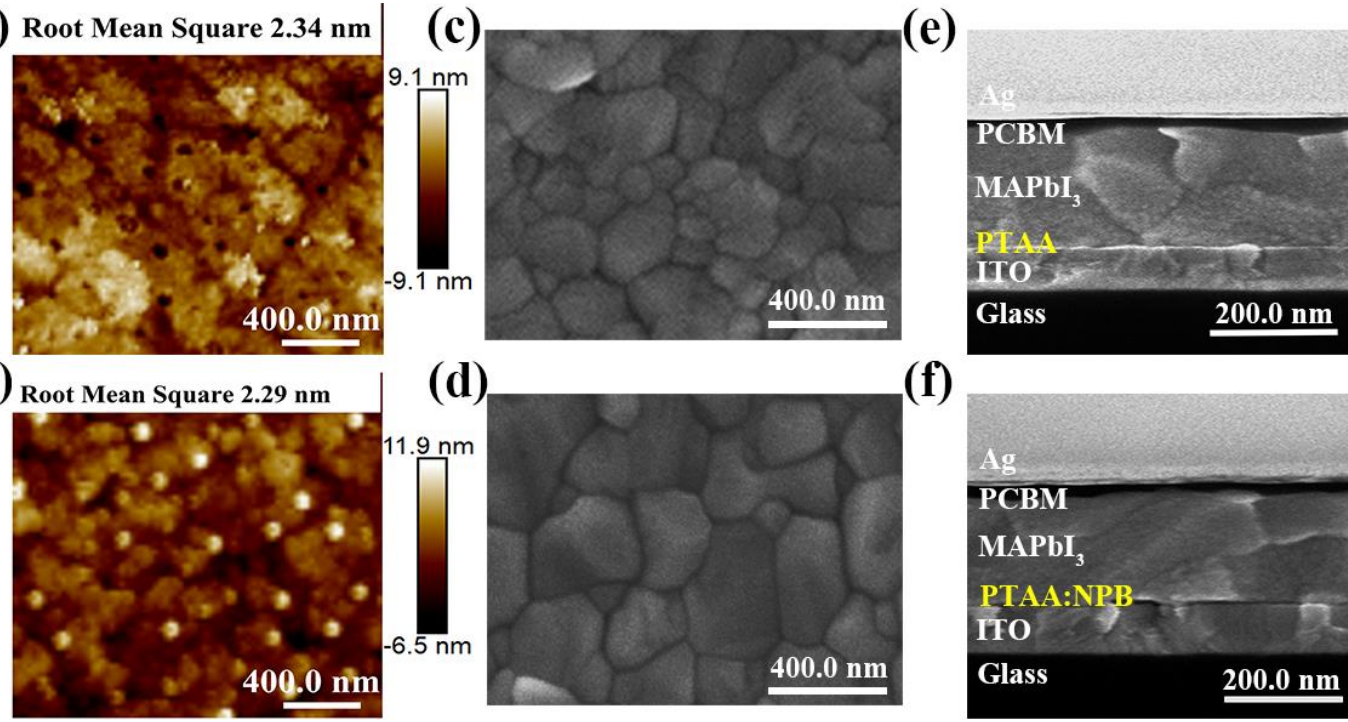

(f)

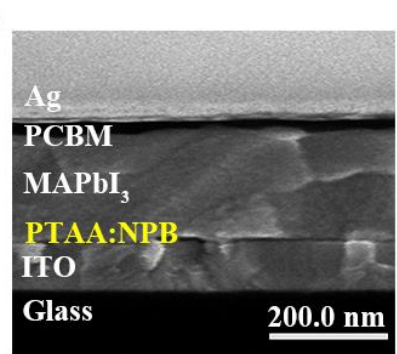

Fig. 2. (a-b) AFM images $(2 \times 2 \mu \mathrm{m})$ of HTLs deposited on ITO, (c-d) Top SEM images of perovskite films deposited on different HTL substrates, (e-f) Cross-sectional view of a perovskite cell in the configuration of 
ITO/HTL/ $\mathrm{CH}_{3} \mathrm{NH}_{3} \mathrm{PbI}_{3} / \mathrm{PC}_{61} \mathrm{BM} / \mathrm{Ag}$ subject to a different PTAA treatment. The corresponding PTAA treated with oxygen plasma and doped with $1.0 \mathrm{wt} \% \mathrm{NPB}$, respectively.

The surface roughness of HTLs with varing NPB wt\% doping was measured using atomic force microscopy (AFM). With $1.0 \mathrm{wt} \%$ of NPB in PTAA, the surface roughness of the HTL is found to be slightly decreased from $2.34 \mathrm{~nm}$ to $2.29 \mathrm{~nm}$ compared to pristine PTAA film with plasma treatment. This indicated that the surface roughness results for doping with the optimum NPB wt\% are similarly to those obtained for plasma treatment. The results also distinctly showed the presence of pinholes in the pristine PTAA film even after oxygen plasma treatment (Fig. S3). Due to the strong apolar nature of PTAA and NPB, a rise in the number of PTAA:NPB aggregates was noted in the NPB-doping PTAA HTL films, leading to a gradual decrease in the number of pinholes with increasing NPB wt\% [35]. It is therefore plausible that the pinholes were filled by aggregates in pristine PTAA, resulting in a decrease in roughness with increasing in NPB wt $\%$. Meanwhile, at $1.5 \mathrm{wt} \%$ of NPB, the PTAA:NPB aggregates in the HTL achieved a high-aggregation state, with no pinholes present in PTAA film to be filled by the aggregates, leading to excessive accumulation of aggregates (protrusions), and hence increased surface roughness. The change in the surface roughness with varying NPB wt\% doping suggested that NPB was successfully doped into PTAA HTLs.

To investigate the impact of NPB doping in PTAA on the morphology of subsequently deposited perovskite films, scanning electron microscopy (SEM) images were studied. Due to the hydrophobic nature of untreated PTAA films, severe dewetting of the precursor solution was observed, resulting in poor film quality. 
Pristine PTAA films with 10 s of oxygen plasma treatment were used as reference for comparative study. Optical images of the corresponding perovskite films were displayed in Fig. S4. It can be found that NPB doping increases the wettability of PTAA films and leads to significantly improved film quality for the perovskite absorber layer, yielding a better film morphology with large grain size (Fig. S5) than that obtained by oxygen plasma treatment (Fig. S6). At $1.0 \mathrm{wt} \%$ of NPB doping, a uniform and smooth perovskite film was formed with a large grain size (Fig. 2d). It appears that the thickness of the HTL was not affected by the NPB doping, as shown in Fig. 2e-f. Moreover, the cross-sectional SEM images further confirm the effect discussed above, i.e., observation of a smooth surface, less pinholes and larger perovskite grains, indicating suppressed grain boundary traps, thus improved device performance [36]. Fewer interface voids and pinholes means a reduction in the carrier recombination area, which will be discussed later [37]. Hence, doping NPB into PTAA appears to facilitate the perovskite crystal growth and increase the grain size by improving the surface wettability of PTAA HTLs. This effect could be attributed to the $\pi$-conjugated nature of NPB, which can optimize interfacial contact and improve perovskite crystal growth, as shown in Fig. 1c. Relating the PTAA:NPB aggregates as mentioned above, the aggregates can act as the growing sites for perovskite crystal nuclei, so the doping of NPB can realize uniform perovskite heterogeneous nucleation and facilitate perovskite growth as well $[38,39]$. 

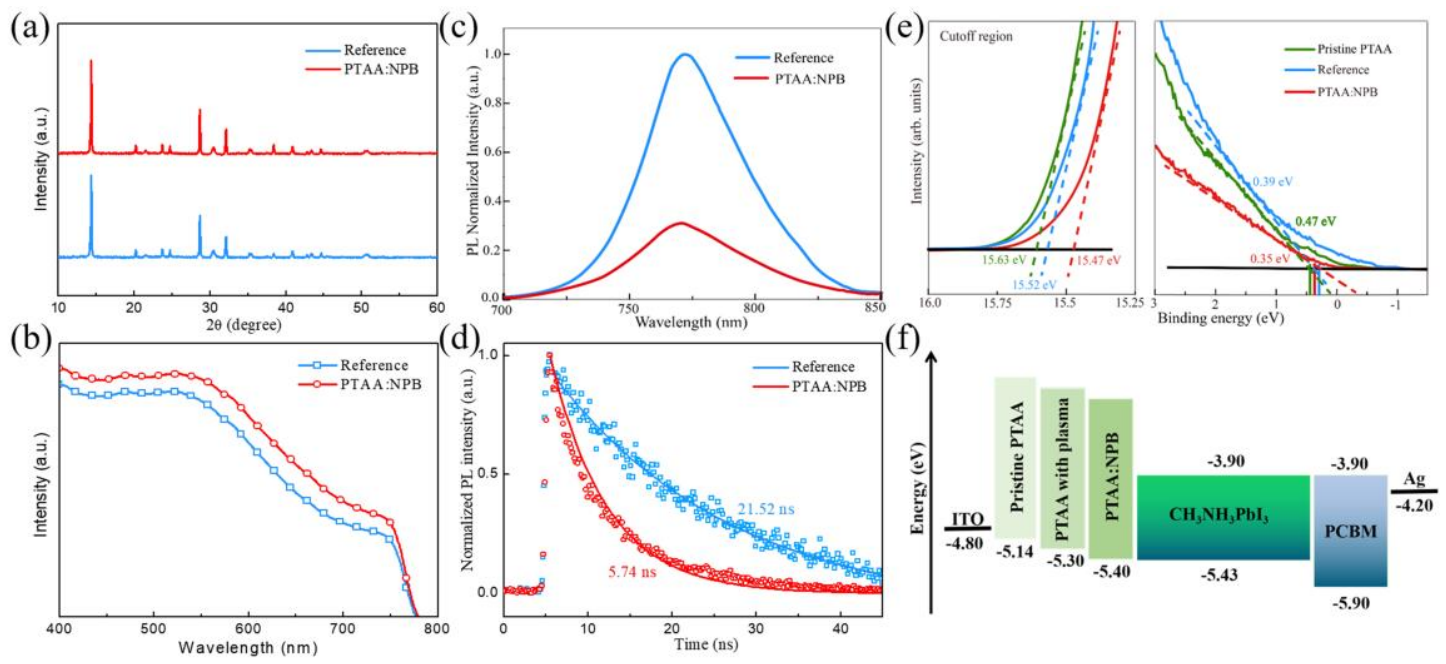

Fig. 3. (a) XRD patterns, (b) Absorption spectra, (c) Steady-state PL spectra, (d) TRPL spectra, and (e) UPS spectra for perovskite films deposited onto ITO/PTAA and ITO/PTAA:NPB substrates. (f) Schematic energy level diagram of the fabricated device with pristine PTAA, with oxygen plasma treated PTAA and PTAA doped with NPB.

The impact of NPB doping on the crystallinity and optical properties of perovskite films was studied using X-ray diffraction (XRD) and ultraviolet-visible (UV-Vis) absorption spectroscopy. Fig. 3a shows the crystallization of $\mathrm{CH}_{3} \mathrm{NH}_{3} \mathrm{PbI}_{3}$ perovskite deposited onto PTAA HTLs with different treatments. The diffraction peaks located at $14.2^{\circ}$ and $28.4^{\circ}$ correspond to the (110) and (220) planes of the tetragonal crystal structure of $\mathrm{CH}_{3} \mathrm{NH}_{3} \mathrm{PbI}_{3}$ [40,41]. With 1.0 wt\% of $\mathrm{NPB}$, the diffraction peaks showed a significant enhancement, indicating that the crystallinity of the perovskite films was improved. Further increase in NPB wt\% leads to a slight decrease in the perovskite diffraction peaks (Fig. S7). It therefore appears that doping NPB in PTAA HTLs with a suitable wt\% plays a positive role in the crystallinity of perovskite films without affecting the crystal structure. Perovskite films deposited onto PTAA HTLs with $1.0 \mathrm{wt} \%$ of NPB exhibit the highest absorbance near the visible light region as revealed in Fig. 3b, consistent with a strengthened crystallinity 
and reduced reflection. Stronger crystallinity also means fewer surface defects and is advantageous in terms of device performance (Fig. S8). Steady-state photoluminescence (PL) and time-resolved PL (TRPL) measurements were used to investigate the influence of NPB doping in PTAA on the carrier extraction and recombination. As shown in Fig. 3c, for the perovskite film deposited onto PTAA:NPB HTL, the PL intensity is clearly weaker than the reference sample, suggesting that surface trap states were passivated for perovskite films deposited onto NPB-doped PTAA HTLs [42-45]. As shown in Fig. 3d, perovskite film deposited onto an NPB-doped PTAA HTL shows a shorter TRPL decay lifetime (5.74 ns) compared to the reference $(21.52 \mathrm{~ns})$. The results suggest that NPB doping can lead to lower defect concentration and efficient charge transfer, thereby leading to improved FF and PCE [46-50]. The electronic structure of the HTLs was studied using UPS, as shown in Fig. 3e. From the secondary electron cutoff spectrum, for pristine PTAA, oxygen plasma treated PTAA and NPB doped in PTAA the work functions were calculated to be 5.59, 5.70 and $5.75 \mathrm{eV}$ and the HOMO energy levels were determined to be 5.12, 5.31 and $5.40 \mathrm{eV}$, respectively. The energy band alignment is illustrated in Fig. 3f. The conduction band minimum (VBM) for $\mathrm{CH}_{3} \mathrm{NH}_{3} \mathrm{PbI}_{3}$ is lower-lying than the HOMO levels of pristine PTAA and plasma-treated PTAA, while the HOMO energy level for NPB doped PTAA HTL is better aligned with the perovskite layer, resulting in a higher $V_{O C}$ for the PSCs $[20,51,52]$. 

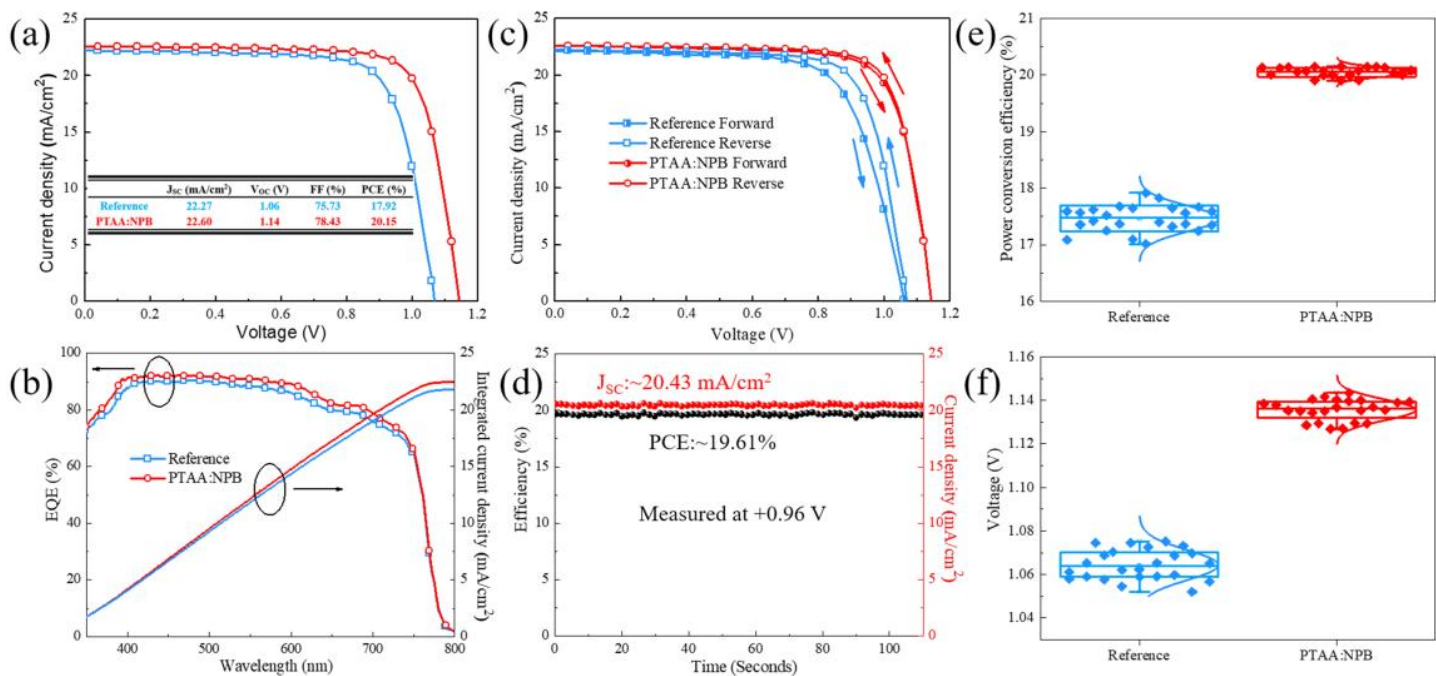

Fig. 4. (a) Best $J-V$ scan curves, (b) EQE spectra, and (c) $J$ - $V$ curves measured under reverse and forward voltage scanning for reference and PTAA:NPB-based devices. (d) Steady-state out profile for the best performance of PTAA:NPB-based PSCs under continuous illumination and a bias voltage of 0.96 V. (e-f) Standard box plot of the PCE and $V_{\mathrm{OC}}$ for the reference and PTAA:NPB-based devices, respectively.

To understand the role of NPB doping on device performance, the best photocurrent density-voltage $(J-V)$ curves are presented in Fig. 4a. The reference device based on oxygen plasma treated PTAA as the HTL showed a PCE of $17.92 \%$ (average $17.46 \%), V_{\mathrm{OC}}$ of $1.06 \mathrm{~V}$, short-circuit current density $\left(J_{\mathrm{SC}}\right)$ of $22.27 \mathrm{~mA} / \mathrm{cm}^{2}$, and FF of $75.7 \%$. On the other hand, the modified device with $1.0 \mathrm{wt} \%$ of NPB in PTAA as the HTL showed a PCE of $20.15 \%$ (average $20.05 \%$ ), $V_{\text {OC }}$ of $1.14 \mathrm{~V}, J_{\mathrm{SC}}$ of $22.60 \mathrm{~mA} / \mathrm{cm}^{2}$, and FF of $78.4 \%$. The details for the devices based on PTAA:NPB with varying wt\% of NPB in PTAA are reported in Table S2 and Fig. S9. The external quantum efficiency (EQE) spectra for the two PSCs are shown in Fig. 4b. The integrated $J_{\mathrm{SC}}$ for the corresponding devices was determined from the EQE to be 21.79 and $22.47 \mathrm{~mA} / \mathrm{cm}^{2}$, respectively, matching the results from $J-V$ characterization. The enhancement of the PSC performance can be attributed to the increased $V_{\mathrm{OC}}$ and FF. 
The hysteresis index (HI) is an important parameter that can be used to quantify the hysteresis loss from $J-V$ curves, it can be measured using both the reverse and forward scan directions shown in Fig. 4c. The HI for devices is defined as follows

$$
H I=\frac{P C E_{\text {reverse }}-P C E_{\text {forward }}}{P C E_{\text {reverse }}}
$$

The HI measured for PTAA:NPB-based PSCs decreased to 0.02 compared with the reference devices (0.07) [48]. Hysteresis is strongly dependent on the contact condition and defects between the perovskite layer and HTLs [53]. As aforementioned, doping NPB in PTAA played a significant role in passivating the perovskite film and interfacial defects. Hence, the modified devices showed a virtually negligible hysteresis compared to the reference device, demonstrating the reliability of the modified devices [40,48].

Moreover, the steady-state photocurrent and PCE were measured at the maximum power output point $(0.96 \mathrm{~V})$ for a PTAA:NPB-based device, as shown in Fig. 4d. The current density and PCE are stabilized at $20.43 \mathrm{~mA} / \mathrm{cm}^{2}$ and $19.61 \%$, respectively, agreeing well with that measured from $J-V$ scanning and confirming the accurate characterization of the device efficiency. To investigate the reproducibility of the PSCs, a standard box plot for the reference and modified devices was drawn. As exhibited in Fig. 4e-f, a conspicuous enhancement of the PCE and $V_{\mathrm{OC}}$ was observed for the devices based on NPB doped PTAA compared to the reference devices. This means that the experimental accidental errors can be excluded, and that the modified devices can demonstrate excellent reproducibility and favorable performance. When the NPB doping increased to 1.5 and $3.0 \mathrm{wt} \%$, the PCE of the corresponding devices 
decreased to $18.20 \%$ (average $17.76 \%$ ) and $16.76 \%$ (average $16.34 \%$ ), respectively (Fig. S9). Therefore, the following characterization was based on PSCs with an optimum NPB doping of $1.0 \mathrm{wt} \%$ in PTAA.
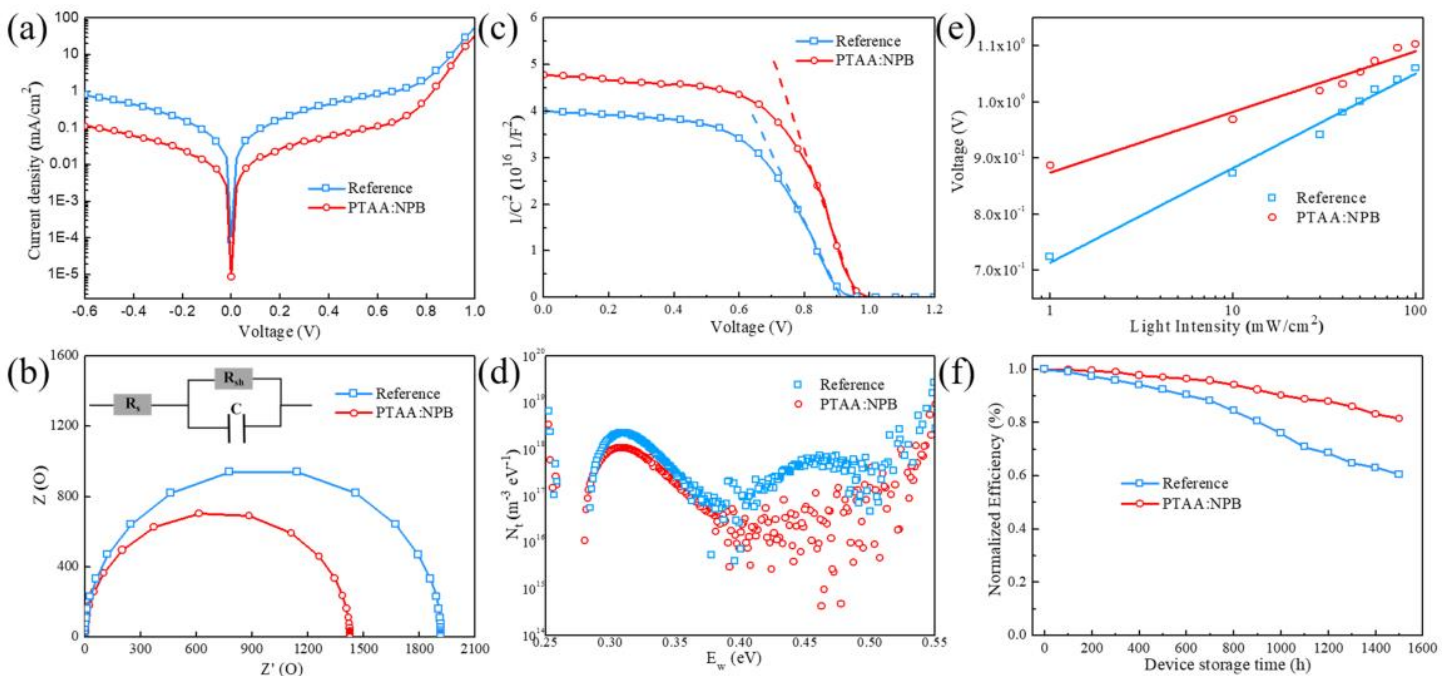

Fig. 5. (a) Dark current characteristics, (b) Nyquist plots measured in the dark at $0.75 \mathrm{~V}$, (c) Mott-Schottky plots measured under dark conditions, (d) Trap density of states (tDOS), and (e) Voc versus light intensity for the reference and PTAA:NPB-based PSCs. (f) Long-term stability of unencapsulated reference and PTAA:NPB-based devices under in ambient air conditions $\left(25^{\circ} \mathrm{C}, 20 \%<\mathrm{RH}<50 \%\right)$.

It is well known that the $V_{O C}$ of a device is mainly dependent on the carrier recombination, which occurs at and across the interface between absorber layer and charge transporting layers $[19,54-57]$. Related to that, we will explain in detail the reason for the observed improvement in $V_{\mathrm{OC}}$ based on carrier separation, transportation and recombination using the dark $J-V$, Mott-Schottky, admittance, and light-intensity-dependent $J-V$ measurements. From Fig. 5a, the dark current density of the PTAA:NPB-based device is clearly lower than that of the reference, explaining why the current leakage can be efficiently restrained through doping NPB into PTAA to a certain extent. It also indicates that carriers are rarely lost when passing the cell, other than direct shunting from the electronic transmission channels $[28,42,44]$. The 
current leakage is mainly dependent on the recombination of carriers, thus leading to the PCE enhancement. The recombination loss in PSCs is also important for determining $V_{O C}$, according to

$$
V_{O C}=\left(\frac{\mathrm{AK}_{\mathrm{B}} \mathrm{T}}{\varepsilon}\right) \ln \left(\frac{J_{S C}}{J_{0}}+1\right)
$$

where $K_{\mathrm{B}}$ is the Boltzmann constant, $T$ is the absolute temperature, and $J_{0}$ is the reverse saturation current density related to the dark current density [58]. PTAA:NPB-based devices possessed a much lower $J_{0}$ compared to the reference, and a lower $J_{0}$ yields a larger $V_{\mathrm{OC}}$ in the device. To clarify the effect of NPB doping on the charge-transport properties of devices, electrochemical impedance spectroscopy (EIS) was performed in the dark with a bias voltage at $0.75 \mathrm{~V}$. The Nyquist plots for the reference and PTAA:NPB-based devices are depicted in Fig. 5b. It was distinctly found that the data showed a semicircular diameter for the modified device that was clearly smaller relative to the reference device, suggesting that charge can be extracted and transported from the absorber layer to HTL more efficiently $[37,59]$. This coincide with the results from the top and cross-sectional SEM images that grain boundary traps in perovskite film decreased after NPB doped into PTAA.

Mott-Schottky (M-S) analysis via capacitance-voltage measurements was performed to explain the role of different HTLs on the $V_{\mathrm{OC}}$ of inverted PSCs. The model is described by the following formula

$$
\frac{1}{C^{2}}=\frac{2}{{\mathrm{q} \varepsilon \mathrm{A}^{2} \mathrm{~N}_{D}}}\left(V_{b i}-V\right)
$$


where $q$ is the elementary charge, $V$ is the applied forward bias, and $A$ is the Schottky contact area, $V_{\text {bi }}$ is the built-in potential, and $N_{D}$ is the carrier density [60]. As shown in Fig. 5c, the value of $V_{b i}$ was $0.90 \mathrm{~V}$ and $0.98 \mathrm{~V}$ for the reference device and PTAA:NPB-based device, respectively. Larger built-in potential means an enhanced driving force for the separation of photo-generated carriers and an extended depletion region for the efficient suppression of holes [61]. Then, the energetics profile for the trap distribution in devices was further discussed through admittance measurements, the angular frequency dependent capacitance (Fig. S10) can be described using the following formula

$$
N_{\mathrm{t}}=-\frac{U_{b i}}{q W_{d}} \bullet \frac{d C}{d \omega} \bullet \frac{\omega}{\mathrm{K}_{\mathrm{B}} \mathrm{T}}
$$

where $C, \omega, U_{\mathrm{bi}}$ and $W_{\mathrm{d}}$ is the capacitance, angular frequency, built-in potential and depletion width, respectively, extracted from the corresponding M-S plot $[62,63]$. As displayed in Fig. 5d, the PTAA:NPB-based device shows almost a one order of magnitude lower trap density compared to the reference device, which symbolizes that NPB doping in PTAA enormously reduces the overall trap states located above the valence band, and thus enhances $V_{\mathrm{OC}}$ and FF $[64,65]$.

To further clarify the reduction of charge recombination caused by NPB doping in PTAA, we carried out light-intensity-dependent $J-V$ measurements. The dependence of $V_{\mathrm{OC}}$ on light intensity in the different HTLs-based cells is depicted in Fig. 5e. The corresponding detailed $J$ - $V$ curves are displayed in Fig. S11. The slope for the PTAA:NPB-based devices $\left(0.11 K_{\mathrm{B}} T / q\right)$ was smaller than that obtained for the reference devices $\left(0.17 K_{\mathrm{B}} T / q\right)$. The smaller slope illustrates the fact that doping NPB 
effectively suppresses trap-assisted charge recombination, which is also in agreement with the conclusion above that NPB doping results in a reduced recombination loss and increased charge lifetimes, thereby contributing to improved device efficiency $[66,67]$.

The device stability was also studied, and the results are depicted in Fig. 5f, the device stability was enhanced when NPB was doped into the HTLs. The stability test result for unencapsulated devices showed that modified device retained approximately $80 \%$ of its initial efficiency compared to $60 \%$ for the reference device after exposure in air for 2 months under common storage conditions (in atmosphere, at room temperature, with humidity in the range of $20 \%-50 \%)$. One reason for this is the strong attraction of $\mathrm{MA}^{+}$to the $\pi$ face of benzene and other aromatic structures, and this strong attraction is enough to restrict the movement of organic cations. Therefore, doping NPB into PTAA can efficiently inhibit the migration of intrinsic ions and consequently, improve the device stability. Another reason for the enhanced stability is that doping NPB in HTL adjusted the wettability of PTAA, this approach improved crystallinity of perovskite film with large grain size and less grain boundary, which are expected to extend device storage time [68]. The stability of the PCE, $J_{\mathrm{SC}}$ and FF values as a function of time are shown in Fig. S12, the $V_{O C}$ remains in the range of $1.12 \mathrm{~V}$. The terminal PCE degradation mainly arises from the sharp decrease in $J_{S C}$ and FF, and this decrease was mainly due to the peeling off of the back electrode Ag after multiple measurements.

\section{Conclusion}


In summary, $\pi$-conjugated small molecule NPB doped into PTAA was demonstrated to serve as an efficient HTL in inverted PSCs, achieving a maximum PCE of $20.15 \%$, along with substantially improved stability compared to pristine PTAA with oxygen plasma treatment. This is because NPB doping increases the hydrophilicity of PTAA and adjusts the HOMO energy level of the HTL to be better aligned with the perovskite valence band. The $\pi$-conjugated nature of NPB leads to stronger cation- $\pi$ interactions with $\mathrm{MA}^{+}$, inducing perovskite crystal growth with larger grain size and better crystallinity. Moreover, PTAA doped with NPB exhibits more efficient hole extraction and carrier transport performance, leading to an increased $V_{O C}$ and FF. This work highlights the potential of NPB doped PTAA as an efficient HTL in inverted PSCs and represents a simple and efficient method to further advance the photovoltaic performance of inverted devices.

\section{Supporting Information}

Supporting Information is available from the Wiley Online Library or from the author.

\section{Acknowledgements}

This work was supported by the National Natural Science Foundation of China (No. 51502081; G11747069), Science and Technique Program of Henan Province (No. 182102210375), Henan Provincial Basic and Frontier Project (No. 152300410088), Henan Provincial Key Science and Technology Research Projects (No. 192102210173) 
and Key Scientific Research Projects of Henan Education Department (NO. 20A140017). This project is also funded by the Collaborative Innovation Center of

Suzhou Nano Science and Technology, and by the Priority Academic Program Development of Jiangsu Higher Education Institutions (PAPD). And the International Postdoctoral Exchange Fellowship Program between Helmholtz-Zentrum Berlin für Materialien und Energie GmbH, OCPC and Soochow University (Chinese home organization).

\section{Reference}

[1] National Renewable Energy Laboratory, Best research-cell efficiencies, https://www.nrel.gov/ncpv/images/efficiencyc_chart.jpg (accessed:August 2019).

[2] J.-Y. Jeng, Y.-F. Chiang, M.-H. Lee, S.-R. Peng, T.-F. Guo, P. Chen, T.-C. Wen, Adv. Mater. 25 (2013) 3727-3732.

[3] T. Salim, S. Sun, Y. Abe, A. Krishna, A. C. Grimsdale, Y. M. Lam, J. Mater. Chem. A 3 (2015) 8943-8969.

[4] M. Liu, M. B. Johnston, H. J. Snaith, Nature 501 (2013) 395-403.

[5] Y. Shao, Z. Xiao, C. Bi, Y. Yuan, J. Huang, Nat. Commun. 5 (2014) 5784-5791.

[6] T. M. Schmidt, T. T. Larsen-Olsen, J. E. Carlé, D. Angmo, F. C. Krebs, Adv. Energy Mater. 5 (2015) 1500569-1500578.

[7] D. Luo, L. Zhao, J. Wu, Q. Hu, Y. Zhang, Z. Xu, Y. Liu, T. Liu, K. Chen, W. Yang, W. Zhang, R. Zhu, Q. Gong, Adv. Mater. 29 (2017) 1604758-1604767.

[8] Y. Bai, X. Meng, S. Yang, Adv. Energy Mater. 8 (2018) 1701883-1701897. 
[9] C. Zuo, L. Ding, Small 11 (2015) 5528-5532.

[10]S. Ye, W. Sun, Y. Li, W. Yan, H. Peng, Z. Bian, Z. Liu, C. Huang, Nano Lett. 15 (2015) 3723-3728.

[11]W. Chen, Y. Zhou, L. Wang, Y. Wu, B. Tu, B. Yu, F. Liu, H. W. Tam, G. Wang, A. B. Djurisic, L. Huang, Z. He, Adv. Mater. 30 (2018) 1800515-1800524.

[12]P. Docampo, J. M. Ball, M. Darwich, G. E. Eperon, H. J. Snaith, Nat. Commun. 4 (2013) 2761-2767.

[13]O. Malinkiewicz, A. Yella, Y. H. Lee, G. M. Espallargas, M. Graetzel, M. K. Nazeeruddin, H. J. Bolink, Nat. Photon. 8 (2013) 128-132.

[14]C. Zuo, L. Ding, Adv. Energy Mater. 7 (2017) 1601193-1601199.

[15]N. J. Jeon, H. Na, E. H. Jung, T.-Y. Yang, Y. G. Lee, G. Kim, H.-W. Shin, S. Il Seok, J. Lee, J. Seo, Nat. Energy 3 (2018) 682-689.

[16]J. Rivnay, S. Inal, B. A. Collins, M. Sessolo, E. Stavrinidou, X. Strakosas, C. Tassone, D. M. Delongchamp, G. G. Malliaras, Nat. Commun. 7 (2016) $11287-11296$.

[17]M. Jørgensen, K. Norrman, F. C. Krebs, Sol. Energy Mat. Sol. C. 92 (2008) 686-714

[18]H.-L. Yip, A. K. Y. Jen, Energy Environ. Sci. 5 (2012) 5994-6011.

[19]S. Zhang, M. Stolterfoht, A. Armin, Q. Lin, F. Zu, J. Sobus, H. Jin, N. Koch, P. Meredith, P. L. Burn, ACS Appl. Mater. Interfaces 10 (2018) 21681-21687.

[20]Y. Kim, E. H. Jung, G. Kim, D. Kim, B. J. Kim, J. Seo, Adv. Energy Mater. 8 (2018) 1801668-1801677. 
[21]W. S. Yang, B.-W. Park, E. H. Jung, N. J. Jeon, Y. C. Kim, D. U. Lee, S. S. Shin, J. Seo, E. K. Kim, J. H. Noh, Science 356 (2017) 1376-1379.

[22]X. Zheng, B. Chen, J. Dai, Y. Fang, Y. Bai, Y. Lin, H. Wei, Xiao C. Zeng, J. Huang, Nat. Energy 2 (2017) 17102-17111.

[23]Y. Deng, Q. Dong, C. Bi, Y. Yuan, J. Huang, Adv. Energy Mater. 6 (2016) $1600372-1600378$.

[24]X. Xu, C. Ma, Y. Cheng, Y.-M. Xie, X. Yi, B. Gautam, S. Chen, H.-W. Li, C.-S. Lee, F. So, J. Power Sources 360 (2017) 157-165.

[25]J. Lee, H. Kang, G. Kim, H. Back, J. Kim, S. Hong, B. Park, E. Lee, K. Lee, Adv. Mater. 29 (2017) 1606363-1606371.

[26]Q. Wang, C. Bi, J. Huang, Nano Energy 15 (2015) 275-280.

[27]X. Liu, Y. Cheng, C. Liu, T. Zhang, N. Zhang, S. Zhang, J. Chen, Q. Xu, J. Ouyang, H. Gong, Energy Environ. Sci. 12 (2019) 1622-1637.

[28]F. Zhang, J. Song, R. Hu, Y. Xiang, J. He, Y. Hao, J. Lian, B. Zhang, P. Zeng, J. Qu, Small 14 (2018) 1704007-1704017.

[29]Y. Li, M. D. Cole, Y. Gao, T. Emrick, Z. Xu, Y. Liu, T. P. Russell, ACS Appl. Energy Mater. 2 (2019) 1634-1641.

[30]N. Li, S. Oida, G. S. Tulevski, S. J. Han, J. B. Hannon, D. K. Sadana, T. C. Chen, Nat. Commun. 4 (2013) 2294-2301.

[31]S. C. Tse, K. C. Kwok, S. K. So, Appl. Phys. Lett. 89 (2006) 262102-262105.

[32]Y. Li, M. D. Cole, Y. Gao, T. Emrick, Z. Xu, Y. Liu, T. P. Russell, ACS Appl. Energy Mater. 2 (2019) 1634-1641. 
[33]W. Nie, J. C. Blancon, A. J. Neukirch, K. Appavoo, H. Tsai, M. Chhowalla, M. A. Alam, M. Y. Sfeir, C. Katan, J. Even, S. Tretiak, J. J. Crochet, G. Gupta, A. D. Mohite, Nat. Commun. 7 (2016) 11574-11583.

[34]C. Duc, A. Vlandas, G. G. Malliaras, V. Senez, Soft Matter 12 (2016) 5146-5153.

[35]S. Masi, A. Rizzo, F. Aiello, F. Balzano, G. Uccello-Barretta, A. Listorti, G. Gigli, S. Colella, Nanoscale 7 (2015) 18956-18963.

[36]J. M. Ball, A. Petrozza, Nat. Energy 1 (2016) 16149-16162.

[37]T. Bu, L. Wu, X. Liu, X. Yang, P. Zhou, X. Yu, T. Qin, J. Shi, S. Wang, S. Li, Z. Ku, Y. Peng, F. Huang, Q. Meng, Y.-B. Cheng, J. Zhong, Adv. Energy Mater. 7 (2017) 1700576-1700586.

[38]Y.-H. Lou, M. Li, Z.-K. Wang, Appl. Phys. Lett. 108 (2016) 053301-053306.

[39]J. Li, M. Zhao, C. Zhao, H. Jian, N. Wang, L. Yao, C. Huang, Y. Zhao, T. Jiu, ACS Appl. Mater. Interfaces 11 (2019) 2626-2631.

[40]J. Li, T. Jiu, S. Chen, L. Liu, Q. Yao, F. Bi, C. Zhao, Z. Wang, M. Zhao, G. Zhang, Y. Xue, F. Lu, Y. Li, Nano Lett. 18 (2018) 6941-6947.

[41]L.-L. Jiang, Z.-K. Wang, M. Li, C.-C. Zhang, Q.-Q. Ye, K.-H. Hu, D.-Z. Lu, P.-F. Fang, L.-S. Liao, Adv. Funct. Mater. 28 (2018) 1705875-1705883.

[42]M. Hou, H. Zhang, Z. Wang, Y. Xia, Y. Chen, W. Huang, ACS Appl. Mater. Interfaces 10 (2018) 30607-30613.

[43]C. Sun, Z. Wu, H.-L. Yip, H. Zhang, X.-F. Jiang, Q. Xue, Z. Hu, Z. Hu, Y. Shen, M. Wang, F. Huang, Y. Cao, Adv. Energy Mater. 6 (2016) 1501534-1501544.

[44]Q. Xue, M. Liu, Z. Li, L. Yan, Z. Hu, J. Zhou, W. Li, X. F. Jiang, B. Xu, F. Huang, 
Adv. Funct. Mater. 28 (2018) 1707444-1707452.

[45]J. Jiang, Q. Wang, Z. Jin, X. Zhang, J. Lei, H. Bin, Z.-G. Zhang, Y. Li, S. F. Liu, Adv. Energy Mater. 8 (2018) 1701757-1701766.

[46]Q. Dong, Y. Fang, Y. Shao, P. Mulligan, J. Qiu, L. Cao, J. Huang, Science 347 (2015) 967-970.

[47]S. D. Stranks, G. E. Eperon, G. Grancini, C. Menelaou, M. J. Alcocer, T. Leijtens, L. M. Herz, A. Petrozza, H. J. Snaith, Science 342 (2013) 341-344.

[48] V. M. Arivunithi, S. S. Reddy, V. G. Sree, H.-Y. Park, J. Park, Y.-C. Kang, E.-S. Shin, Y.-Y. Noh, M. Song, S.-H. Jin, Adv. Energy Mater. 8 (2018) $1801637-1801645$.

[49]A. Abate, M. Saliba, D. J. Hollman, S. D. Stranks, K. Wojciechowski, R. Avolio, G. Grancini, A. Petrozza, H. J. Snaith, Nano Lett. 14 (2014) 3247-3254.

[50]N. K. Noel, A. Abate, S. D. Stranks, E. S. Parrott, V. M. Burlakov, A. Goriely, H. J. Snaith, ACS Nano 8 (2014) 9815-9821.

[51]Y. Shao, Y. Yuan, J. Huang, Nat. Energy 1 (2016) 15001-15007.

[52]C. Liu, W. Li, J. Chen, J. Fan, Y. Mai, R. E. Schropp, Nano Energy 41 (2017) $75-83$.

[53]C. H. Chiang, C. G. Wu, ACS Nano 12 (2018) 10355-10364.

[54]J.-P. Correa-Baena, W. Tress, K. Domanski, E. H. Anaraki, S.-H. Turren-Cruz, B. Roose, P. P. Boix, M. Grätzel, M. Saliba, A. Abate, A. Hagfeldt, Energy Environ. Sci. 10 (2017) 1207-1212.

[55]C. M. Wolff, F. Zu, A. Paulke, L. P. Toro, N. Koch, D. Neher, Adv. Mater. 29 
(2017) 1700159-1700167.

[56]N. F. Montcada, J. M. Marín-Beloqui, W. Cambarau, J. Jiménez-López, L. Cabau, K. T. Cho, M. K. Nazeeruddin, E. Palomares, ACS Energy Lett. 2 (2016) 182-187.

[57]S. Wheeler, D. Bryant, J. Troughton, T. Kirchartz, T. Watson, J. Nelson, J. R. Durrant, J. Phys. Chem. C 121 (2017) 13496-13506.

[58]J. Shi, J. Dong, S. Lv, Y. Xu, L. Zhu, J. Xiao, X. Xu, H. Wu, D. Li, Y. Luo, Appl. Phys. Lett. 104 (2014) 063901-063905.

[59]M. Li, Z.-K. Wang, Y.-G. Yang, Y. Hu, S.-L. Feng, J.-M. Wang, X.-Y. Gao, L.-S. Liao, Adv. Energy Mater. 6 (2016) 1601156-1601164.

[60]J. P. Clifford, K. W. Johnston, L. Levina, E. H. Sargent, Appl. Phys. Lett. 91 (2007) 253117-253120.

[61]L. Liu, A. Mei, T. Liu, P. Jiang, Y. Sheng, L. Zhang, H. Han, J. Am. Chem. Soc. 137 (2015) 1790-1793.

[62]T. Walter, R. Herberholz, C. Müller, H. W. Schock, J. Appl. Phys. 80 (1996) 4411-4420.

[63]R. Herberholz, M. Igalson, H. W. Schock, J. Appl. Phys. 83 (1998) 318-325.

[64]Y. Lin, L. Shen, J. Dai, Y. Deng, Y. Wu, Y. Bai, X. Zheng, J. Wang, Y. Fang, H. Wei, W. Ma, X. C. Zeng, X. Zhan, J. Huang, Adv. Mater. 29 (2017) $1604545-1604551$.

[65]S. Wang, P. Kaienburg, B. Klingebiel, D. Schillings, T. Kirchartz, J. Phys. Chem. C 122 (2018) 9795-9803. 
[66]X. Yin, C. Wang, D. Zhao, N. Shrestha, C. R. Grice, L. Guan, Z. Song, C. Chen, C. Li, G. Chi, B. Zhou, J. Yu, Z. Zhang, R. J. Ellingson, J. Zhou, Y. Yan, W. Tang, Nano Energy 51 (2018) 680-687.

[67]T. Singh, T. Miyasaka, Adv. Energy Mater. 8 (2018) 1700677-1700686.

[68]A. Fakharuddin, F. Di Giacomo, I. Ahmed, Q. Wali, T.M. Brown, R. Jose, J. Power Sources, 283 (2015) 61-67.

\section{Highlights}

(1) NPB doping into PTAA enhanced interface contact of HTL and absorber layer.

(2) Perovskite formed on top of PTAA:NPB with better crystallinity and morphology.

(3) Hole extracted and carrier transported more efficiently in modified device.

(4) Over 20\% PCE of planar p-i-n device based on the PTAA:NPB was obtained.

\section{Graphical Abstract}

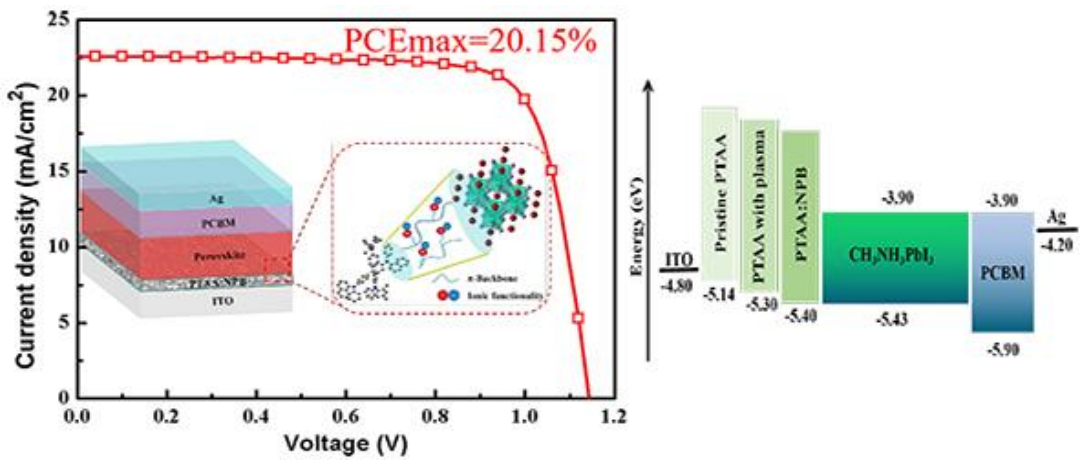

